\title{
12 h Abstinence-Induced ERP Changes in Young Smokers: Electrophysiological Evidence From a Go/NoGo Study
}

\begin{abstract}
Chang Liu't, Fang Dong't, Yangding Li ${ }^{2}$, Yan Ren', Dongdong Xie', Xianfu Wang', Ting Xue ${ }^{1}$, Ming Zhang ${ }^{1}$, Guoyin Ren ${ }^{1}$, Karen M. von Deneen ${ }^{3}$, Kai Yuan ${ }^{1,2,3 *}$ and Dahua Yu ${ }^{1 *}$
\end{abstract}

1 Inner Mongolia Key Laboratory of Pattern Recognition and Intelligent Image Processing, School of Information Engineering, Inner Mongolia University of Science and Technology, Baotou, China, ${ }^{2}$ Guangxi Key Laboratory of Multi-Source Information Mining and Security, Guangxi Normal University, Guilin, China, ${ }^{3}$ School of Life Sciences and Technology, Xidian University, Xi'an, China

OPEN ACCESS

Edited by:

Qinghua He,

Southwest University, China

Reviewed by:

Yuzheng $\mathrm{Hu}$,

Zhejiang University, China Linling Li,

Shenzhen University, China

${ }^{*}$ Correspondence:

Kai Yuan

kyuan@xidian.edu.cn

Dahua Yu

fmydh@imust.edu.cn

${ }^{\dagger}$ These authors have contributed equally to this work

Specialty section:

This article was submitted to

Psychopathology,

a section of the journal

Frontiers in Psychology

Received: 05 May 2019

Accepted: 22 July 2019

Published: 14 August 2019

Citation:

Liu C, Dong F, Li Y, Ren Y, Xie D, Wang $X$, Xue T, Zhang $M$, Ren $G$, von Deneen KM, Yuan K and Yu D (2019) 12 h Abstinence-Induced ERP

Changes in Young Smokers: Electrophysiological Evidence From a Go/NoGo Study.

Front. Psychol. 10:1814. doi: 10.3389/fpsyg.2019.01814
Decreased inhibition control ability and increased craving may be the most important causes of relapsing in smoking. Although inhibition control defects in young smokers were investigated, the effects of short-term abstinence on inhibition control in young smokers were still unclear. Thirty young smokers participated in the present study. The EEG signals during the Go/NoGo task were recorded in both satiety and $12 \mathrm{~h}$ abstinence conditions. The task performances were observed and compared between the two conditions. Event-related potential (ERP) analysis was used to investigate changes in N200 and P300 amplitude and latency induced by $12 \mathrm{~h}$ of abstinence. After $12 \mathrm{~h}$ of abstinence, the latency of N200 was prolonged in young smokers. No significant changes were found in the number of NoGo errors and the response time of Go in young smokers after $12 \mathrm{~h}$ of abstinence. Correlation analysis showed that the N200 latency of abstinence condition was significantly correlated with the number of NoGo errors and the response time of $\mathrm{Go}$ in the abstinence condition. The present findings may improve the understanding of the effect of short-term abstinence in young smokers. We suggested that the latency of N200 may be associated with inefficient inhibitory control of the abstinence condition in young smokers. Our results may contribute new insights into the neural mechanism of nicotine abstinence in young smokers.

Keywords: event-related potentials, young smokers, 12-h abstinence, Go/NoGo task, inhibition control

\section{INTRODUCTION}

Smoking is the leading cause of death in the world, which may cause 6 million people to die annually (World Health Organization, 2013). "China Report on the Health Hazards of Smoking" in 2012 indicated that China had 350 million smokers including 14 million young smokers and more than 1 million deaths each year due to smoking-related diseases. A national survey showed that young adults had the highest smoking rate than any age group in the United States (Ling et al., 2009). In addition, at least $20 \%$ of young smokers which had higher levels of nicotine dependence became regular smokers (Lantz, 2003). Previous studies of young smokers reported that nicotine 
may affect brain maturation and nervous system development in adolescents (Galván et al., 2011; Li et al., 2015; Bu et al., 2016; Yuan et al., 2016a, 2017, 2018; Su et al., 2017; Wang et al., 2017; Yu et al., 2017a,b; Zhang et al., 2017). Although most young smokers are aware of the negative consequences of smoking and expressed a strong desire to quit smoking, the vast majority of quitting attempts ended in relapse (Kenford et al., 2002). Previous studies found that smokers had nicotine withdrawal symptoms during the withdrawal state as increased craving and decreased cognitive control ability, which may lead to the failure of smoking cessation and even relapse (Jacobsen et al., 2004; Lerman et al., 2014). On average, the percentage of smokers who successfully quit more than one year will not exceed 5\% (Jamal et al., 2014). The changes of craving and cognitive control during the short-term abstinence state may improve our understanding of smoking for young people (Cahill et al., 2012).

Cognitive impairment is related to nicotine abstinence, which may be an important cause of relapse (Beaver et al., 2011; Yuan et al., 2016b). Previous studies in our group confirmed there were functional changes in the brain caused by 12-habstinence in young smokers ( $\mathrm{Li}$ et al., 2016; Bi et al., 2017; Kai et al., 2018; Zhao et al., 2018). However, there are few studies on brain electrophysiological changes caused by 12 -h abstinence in young smokers. As a reliable method, the utilization of a longitudinal experiment design not only increased statistical power, but also allowed for the examination of correlations between changes in inhibition control, and changes in brain activation across sessions (i.e., satiety vs. smoking abstinence) (Borst et al., 2014; Paulsen et al., 2015). In contrast to fMRI technique measuring hemodynamic response, the EEG/ERP technique captures electrophysiological signals directly related to neuronal activity. Present study mainly explored the changes of inhibitory control and electrophysiological changes induced by $12 \mathrm{~h}$ of abstinence in young smokers.

In the current study, the inhibitory cognitive control ability of young smokers was measured by a Go/NoGo task. In this task, participants responded quickly to "Go" stimuli without responding to "NoGo" stimuli, and the more they responded to "NoGo" stimuli, the more serious their inhibitory cognitive control deficits (Figure 1; Littel et al., 2012). Event-related

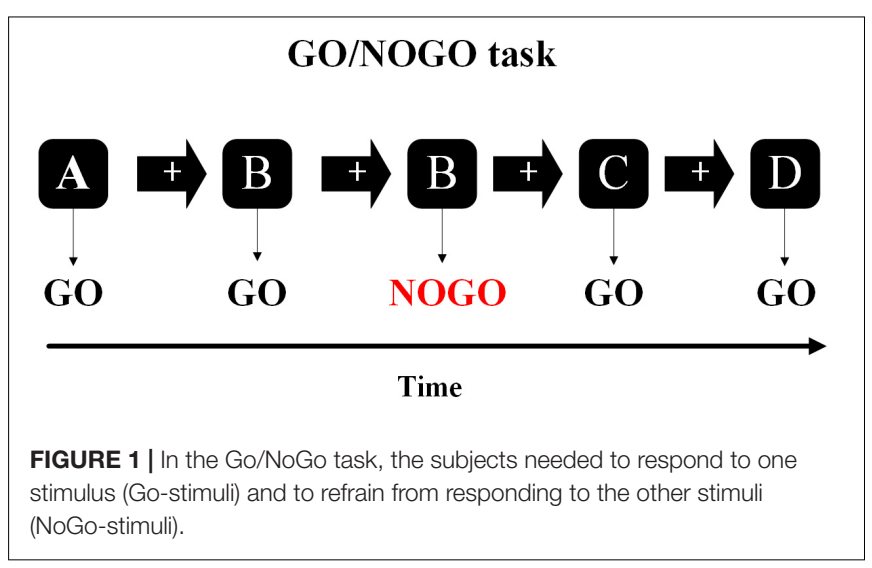

TABLE 1 | Demographic characteristics of young smokers in the present study.

\begin{tabular}{lc}
\hline Clinical details & Young smokers $(\boldsymbol{n}=\mathbf{3 0})$ \\
\hline Age (years) & $20.9 \pm 1.19$ \\
Age range (years) & $19-21$ \\
Education (years) & $14.06 \pm 0.57$ \\
Age of smoking initiation & $15.2 \pm 2.76$ \\
Smoking duration (years) & $4.31 \pm 2.19$ \\
Pack-years & $3.13 \pm 2.49$ \\
Cigarettes per day (CPD) & $14.27 \pm 4.35$ \\
FTND total score & $4.73 \pm 1.59$ \\
\hline
\end{tabular}

Values are expressed as means \pm standard deviations. FTND, Fagerström Test for nicotine dependence; Pack-years, smoking years $\times$ daily consumption/20.

potentials (ERP) were used to investigate the changes of NoGoN200 and NoGo-P300 amplitude and latency of Go/NoGo task between satiety and $12 \mathrm{~h}$ abstinence sessions in young smokers. Two major ERP components, N200 and P300, have been shown to be associated with smoker inhibition control in the Go/NoGo task (Bokura et al., 2001; Luijten et al., 2011; Cid-Fernández et al., 2014). Among them, P300 is a positive wave about $300 \mathrm{~ms}$ after the reaction occurs, which is related to the actual inhibition process (Falkenstein et al., 1999; Cheng et al., 2016). N200 is a negative wave about $200 \mathrm{~ms}$ after the reaction occurs, which is related to the conflict detection during the early stage of the inhibition process (Donkers and van Boxtel, 2004). We hypothesized that the electrophysiological measurements and behavior of young smokers would change after $12 \mathrm{~h}$ of abstinence during the Go/NoGo task, and these changes were related to the inhibition control impairments in young smokers.

\section{MATERIALS AND METHODS}

\section{Participants}

Thirty male smokers (19-21 years of age) were recruited from Inner Mongolia University of Science and Technology in the present study. All smokers had normal vision. The selection criteria for young male smokers who have never quit smoking before were as follows: (1) reported smoking more than 10 cigarettes a day in the last 6 months; (2) met the DSM-V criteria for current nicotine dependence; and (3) no smoking cessation in the past year. Additionally, nicotine dependence levels were assessed with the Fagerstrom Test of nicotine dependence (FTND) (Fagerstrom and Schneider, 1989). (4) Participants were right-handed as measured by the Edinburgh Handedness Inventory (Oldfield, 1971). (5) Had no physical illness or neurological/psychiatric disorders as assessed by clinical evaluations and medical records, or alcohol/drug abuse. All participants were already familiar with the experimental process and signed the written informed consent. More detailed information can be found in Table $\mathbf{1}$.

\section{Procedures}

Participants filled out questionnaires on demographics. We used a within-subject design with two identical EEG sessions 
occurring 1-3 weeks apart: (1) smoking as usual (satiety) and (2) $\geq 12 \mathrm{~h}$ abstinence (abstinence). The smoking satiety participants could smoke within 20 min before the experiment. During abstinence, the participants were banned from using cigarettes, alcohol or other drugs (including caffeine and nicotine) at least $12 \mathrm{~h}$ prior to the experiment. Before starting the experiment, the participants sat comfortably and the electrodes were connected. Participants were told that mistakes are inevitable, but they should do their best to complete the task as quickly and accurately as possible. Then, all participants were asked to complete the Go/NoGo task and their EEG data were collected simultaneously. This study used E-prime 2.0 software (Psychology Tools, Pittsburgh, PA, United States) to collect participants' behavior data. The authenticity of the 12-h abstinence was confirmed by the expiratory carbon monoxide (CO) levels measured using the Smokerlyzer system (Bedfont Scientific Ltd., Rochester, United Kingdom). Specific measurement criteria were that all subjects had to have their CO levels measured $10 \mathrm{~min}$ before the beginning of the experiment. The participants were required to stand up and hold the Smokerlyzer system in their right hand. When the Smokerlyzer system began to detect CO, the participants were asked to breathe out $\mathrm{CO}$ into the Smokerlyzer system with their mouth. CO level in the expired air was verified as $\leq 8$ parts per million (ppm) during the abstinence state, which showed a distinct reduction for each participant compared to that measured during the satiety state (>10 ppm) (Table 2; Heimstra et al., 1980).

\section{Task Paradigm}

The Go/NoGo task was divided into four sections, each consisting of 159 letters (e.g., A, B, C, and D), with $60 \mathrm{~s}$ of rest after each section. About 74 stimulations (NoGo trials) were randomly inserted into the task and the stimulation was never continuous. The letter appears in the middle of the screen for $600 \mathrm{~ms}$, preceded by a white cross against a black ground for $300 \mathrm{~ms}$. Participants were asked to respond with their right index finger quickly to the stimulus (Go trials), but remained silent on the repeated stimulation (NoGo trials) (Yin et al., 2016).

\section{EEG Recording and Data Analysis}

The electroencephalogram (EEG) recordings were performed in a closed, quiet room. The participants were arranged to be $100 \mathrm{~cm}$ away from the screen to ensure that the display was clearly visible. EEG data were recorded by using the BrainAmp MR plus (Brain Products GmbH. Munich. Germany) with the electrodes at 64 scalp sites (positioned following the 1020 International System) with an additional electrode at $\mathrm{FCz}$ (reference electrode). The vertical electrooculogram (EOG) of the two electrodes was recorded at a position above the left eye and at an outer corner of the right eye. All signals were digitalized with a sample rate of $1000 \mathrm{~Hz}$ with a frequency band from 0.10 to $250 \mathrm{~Hz}$, and impedances were reduced to less than $10 \mathrm{k} \Omega$. All offline data were processed using Brain Vision Analyzer 2 (Brain Products GmbH. Munich. Germany). The EEG data re-referenced the mean values of the mastoids (TP9 and TP10). EEG signals were band-pass filtered using a $0.15-30 \mathrm{~Hz}$ (IIR filter $24 \mathrm{~dB}$ /octave roll off, $50 \mathrm{~Hz}$ notch) band-pass filter. Eye movements and eye blinks were removed using an independent component analysis (ICA). Raw data inspection was done for all epochs (-200 ms prestimulus to $800 \mathrm{~ms}$ post-stimulus). The rejection criteria were: maximal allowed voltage step (gradient) was $50 \mu \mathrm{v}$ for each sample point, maximal allowed amplitude was $\sim 75 \sim 75 \mu \mathrm{v}$, maximal allowed value difference was $100 \mu \mathrm{v}$ in a $200 \mathrm{~ms}$ interval and activity below $0.5 \mu \mathrm{v}$ in a $100 \mathrm{~ms}$ interval was rejected. All data were then baseline corrected from -200 to $0 \mathrm{~ms}$ pre-stimulus. Average of the data for a specific epoch was done according to the condition (NoGo). Based on previous studies, the N200 and P300 components were analyzed at PZ and FCz electrode points (Tian and Yao, 2008). The N200 and P300 amplitudes were defined as the global maximal value to baseline at the signal subject level (N200, 150 300 ms post-stimulus; P300, 250 400 ms post-stimulus) (Littel et al., 2012).

TABLE 2 | The age of each smoker and the CO in both sessions (satiety and abstinence).

\begin{tabular}{|c|c|c|c|}
\hline \multirow[t]{3}{*}{ Subject ID } & \multicolumn{3}{|c|}{ Items } \\
\hline & \multirow[t]{2}{*}{ Age (years) } & \multicolumn{2}{|c|}{ CO (ppm) } \\
\hline & & Satiety & Abstinence \\
\hline 1 & 20 & 11 & 7 \\
\hline 2 & 20 & 13 & 4 \\
\hline 3 & 20 & 10 & 6 \\
\hline 4 & 21 & 11 & 2 \\
\hline 5 & 21 & 10 & 3 \\
\hline 6 & 20 & 12 & 5 \\
\hline 7 & 20 & 18 & 4 \\
\hline 8 & 19 & 12 & 3 \\
\hline 9 & 21 & 14 & 5 \\
\hline 10 & 20 & 11 & 3 \\
\hline 11 & 21 & 12 & 2 \\
\hline 12 & 21 & 14 & 2 \\
\hline 13 & 20 & 13 & 5 \\
\hline 14 & 20 & 16 & 6 \\
\hline 15 & 20 & 13 & 5 \\
\hline 16 & 19 & 15 & 3 \\
\hline 17 & 21 & 12 & 4 \\
\hline 18 & 21 & 14 & 2 \\
\hline 19 & 19 & 11 & 5 \\
\hline 20 & 20 & 10 & 3 \\
\hline 21 & 21 & 12 & 8 \\
\hline 22 & 21 & 15 & 7 \\
\hline 23 & 21 & 12 & 2 \\
\hline 24 & 20 & 12 & 2 \\
\hline 25 & 20 & 19 & 7 \\
\hline 26 & 21 & 12 & 2 \\
\hline 27 & 20 & 13 & 4 \\
\hline 28 & 20 & 15 & 4 \\
\hline 29 & 20 & 10 & 4 \\
\hline 30 & 21 & 11 & 4 \\
\hline
\end{tabular}




\section{Statistics}

Statistical analysis of all data was performed using the Statistical Product and Service Solution 20 (SPSS 20). Two-sample paired $t$-test was used to detect differences in behavioral data (NoGo errors, Go reaction time) and ERP data (amplitude and latency of the N200 and P300) between the satiety session and abstinence session. Pearson correlation coefficients were calculated for behavioral data (NoGo errors, Go reaction time), smoking status [pack-years, FTND, cigarettes per day (CPD)] and both latency, and amplitude of ERPs (N200 and P300).

\section{RESULTS}

\section{EEG Data N200}

After $12 \mathrm{~h}$ of abstinence, the latency of N200 was prolonged compared with that in the satiety sessions during the Go/NoGo task state in young smokers $(t=-2.517, p=0.018)$. The amplitude of N200 in 12-h smoking abstinence condition had an increasing trend compared with the smoking satiety sessions in young smokers but the difference was not significant $(t=-0.904$, $p=0.37$ ) (Figure 2).

\section{P300}

There was no significant difference between the smoking satiety group and the 12-h smoking abstinence session on amplitude $(t=-0.851, p=0.402)$ and latency $(t=0.307, p=0.716)$ of P300 (Figure 2).

\section{Behavioral Data}

No significant difference was found in the Go response time (RT) (12-habstinence: $380.16 \pm 41.17$, satiety: $377.96 \pm 46.84$, $p=0.84$ ) and NoGo errors (12-h abstinence: $33.20 \pm 11.70$, satiety: $33.93 \pm 10.55 ; p=0.8$ ) between the smoking satiety session and the 12-h smoking abstinence session (Figure 3).

\section{Correlations}

Pearson correlation coefficients were calculated for the behavioral data and smoking status (pack-years, FTND, and CPD) and both latency and amplitude of the NoGoN200 and P300. For the 12-h smoking abstinence session, a significant correlation was found between NoGo errors and N200 latency $(r=-0.487$, $p=0.006$ ), and a significant correlation was also found between the Go response time and N200 latency $(r=-0.472, p=0.008)$ (Figure 4). No significant correlation was found between the electrophysiological measurements and clinical measures, packyears, cigarettes per day, and FTND. Furthermore, a correlation analysis replicated previous results showing a significant correlation between the P300 amplitude and NoGo errors during the smoking satiety session $(r=0.370, p=0.044)$.

\section{DISCUSSION}

Young smokers may become lifelong smokers which will make it difficult to quit smoking in the future. The failure of young smokers to quit smoking is probably related to inhibition control (Lubman et al., 2015). The weakening of the inhibition control ability may make them unable to control their desire to smoke leading to relapse. Studying the critical moment of early smoking abstinence in adolescent smokers, especially $12 \mathrm{~h}$ of smoking abstinence, is likely to help us understand the neurological mechanism of smoking. For example, the latest study in our group found that the tract strength of the left striatum-dorsolateral PFC (DLPFC) during the 12-h abstinence predicted the lapse in smokers with an accuracy of $68.3 \%$ (Kai et al., 2018). Event-related potential (ERP) can accurately detect changes in brain-evoked potential under different events due to its high temporal resolution, providing the flow of information almost in real time (Falkenstein et al., 1999; Gajewski and Falkenstein, 2013; Kamarajan and Porjesz, 2015). Therefore, we employed the ERP method to compare inhibition control ability between 12-h abstinence vs. satiety conditions in young smokers whose ERP components are usually quantified by their amplitude and latency measures. N200 and P300 each reflect unique cognitive brain functions (e.g., attention, motivation, and higher level executive function) (Patel and Azzam, 2005; Parvaz et al., 2011; Gajewski and Falkenstein, 2013). In Go/NoGo tasks, the more NoGo errors, the weaker the inhibition and control ability, while the longer the response time of Go, and the worse the response performance to the task (Luijten et al., 2011; Littel et al., 2012). In the present study, we found that the latency of N200 was prolonged in young smokers after $12 \mathrm{~h}$ of abstinence. In addition, previous research by our group found that young smokers were deficient in the inhibition control ability and the P300 amplitude of young smokers was lower than that of non-smokers (Yin et al., 2016). However, the 12-h abstinence did not make the young smokers' inhibition control deficits more serious. In contrast, another study found that short-term abstinence could lead to reduced heart rate, worse task performance, feelings of depression, stress, irritability, restlessness, poor concentration, and urges to smoke (Parrott et al., 2015). Moreover, the correlation analysis showed that there was a significant correlation between N200 latency and NoGo errors and Go response time after $12 \mathrm{~h}$ of abstinence in young smokers.

It was demonstrated that N200 represented the process of reaction inhibition or conflict monitoring (Donkers and van Boxtel, 2004; Bekker et al., 2005; Kaiser et al., 2006). In the Go/NoGo task, the NoGo-N200 was related to increased effort in activating the response inhibition system that interrupted preparations for response execution (Géczy et al., 1999; Bokura et al., 2001; Tian and Yao, 2008). In addition, related research showed that ERP indices have been used to predict relapse (Parvaz et al., 2011). For example, ERP studies in sober alcoholics found delayed N200 latency to distinguish between abstainers and relapsers with an overall predictive rate of $71 \%$ (Glenn et al., 1993). Furthermore, it has been suggested that N200 latency is an indicator of the rate of inhibition of control response preparation, and is related to cognitive monitoring efficiency (Falkenstein et al., 1999; Gajewski and Falkenstein, 2013). For instance, in a study observing behavioral responses of activation and inhibition 


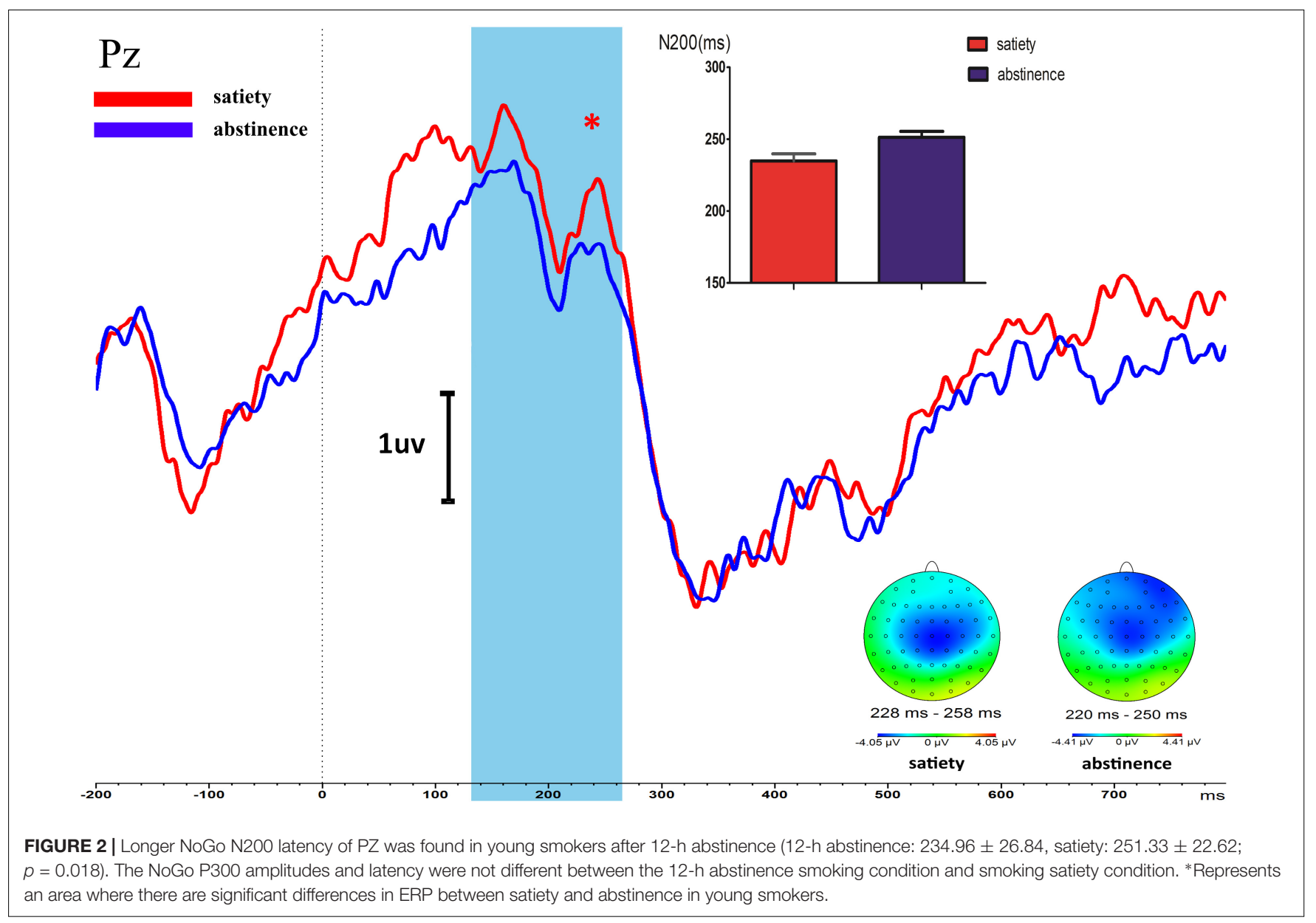

A

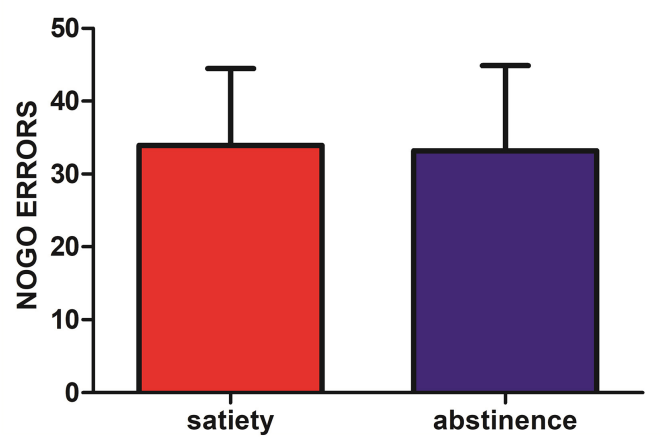

B

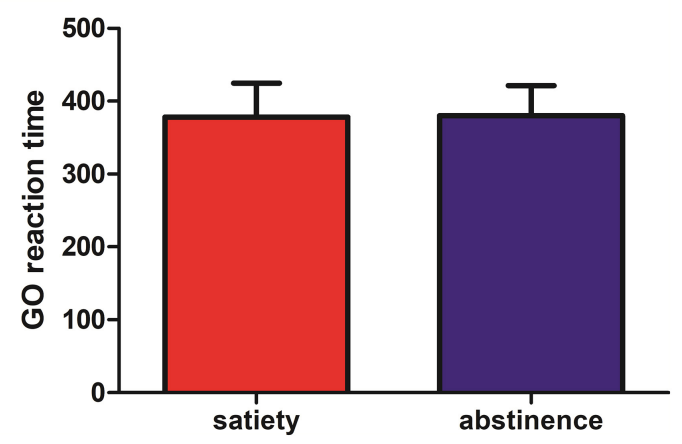

FIGURE 3 | (A) No significant difference was found in NoGo errors (12-h abstinence: $33.20 \pm 11.70$, satiety: $33.93 \pm 10.55 ; p=0.8)$ after 12 -h abstinence. (B) No significant difference was found in Go reaction time (RT) (12-h abstinence: $380.16 \pm 41.17$, satiety: $377.96 \pm 46.84 ; p=0.84)$ after 12 -h abstinence.

processes in children, adolescents, and adult populations, the better the task performance, and the longer the latency of N200 (Johnstone et al., 2005). In another study comparing adolescent smokers with non-smokers, smokers exhibited a reduced N200 component with no behavioral deficits suggesting that N200 may provide a sensitive index of cognitive control deficits in smokers (Buzzell et al., 2014). Therefore, the abnormality of the N200 component, especially the abnormality of the N200 latency, is likely to be regarded as a marker for neural deficits in inhibition control in the abstinence condition. Previous studies in our group suggested that smoking abstinence dramatically alters neural mechanisms underlying interactions, thus affecting the balance between reward and cognitive control (Li et al., 2016; Bi et al., 2017; Kai et al., 2018; 

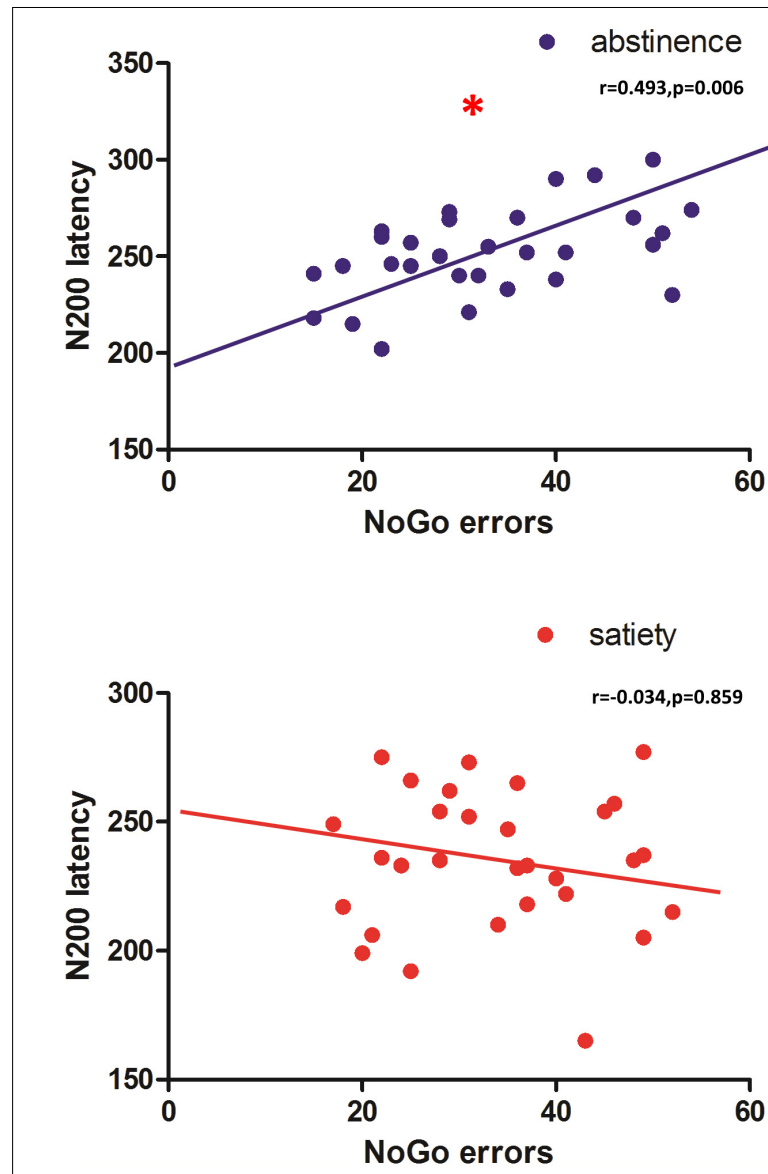
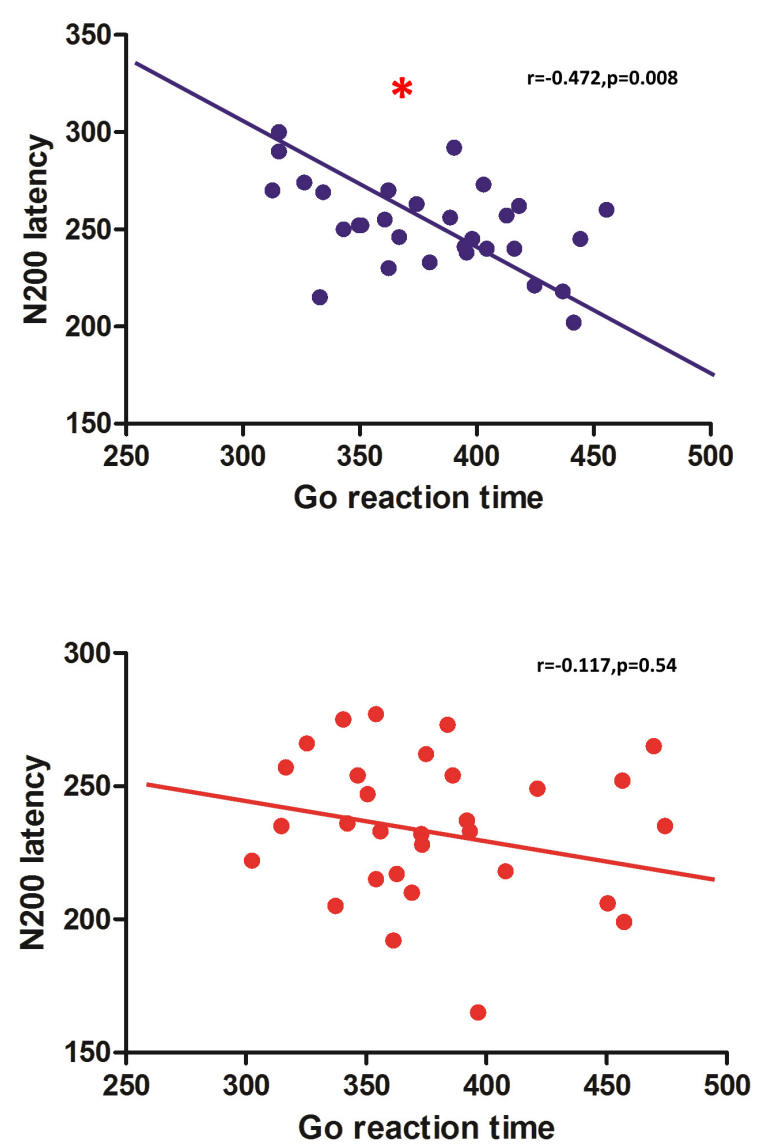

FIGURE 4 | N200 latency of the abstinence condition was significantly correlated with the number of NoGo errors ( $r=0.493, p=0.006)$ and the response time of Go errors $(r=0.472, p=0.008)$. No significant correlation was found in the smoking satiety condition. *Represents that there is a significant correlation between NoGo errors, the response time of Go errors and the N200 latency.

Zhao et al., 2018). In the present study, the result that reduced inhibitory control ability was found in NoGo accuracy as well the N200 component changes of the ERP provides support for the hypothesis that there is a general shortcoming in response inhibition control in young smokers after $12 \mathrm{~h}$ of abstinence. Furthermore, N200 latency was related with NoGo errors during the abstinence condition suggesting that N200 latency may be related to the inhibition control ability in young smokers after $12 \mathrm{~h}$ of abstinence. The correlation between N200 latency and Go reaction time also reflects that N200 latency may be related to reaction performance. However, the current results do not fully support this hypothesis. It may be that reduced inhibitory control ability is the result of prolonged nicotine dependence in young smokers; for example, this may occur via abnormalities in the dopamine system or due to low levels of nicotine dependence (Luijten et al., 2011). In the future, we will further explore the relationship between N200 and inhibition control ability in young smokers after abstinence for $12 \mathrm{~h}$.

P300 is closely related to the actual inhibition process of the reaction (Evans et al., 2010; Littel et al., 2012; Gajewski and Falkenstein, 2013; Cid-Fernández et al., 2014). Consistent with our previous studies, the present study found that the P300 amplitude of young smokers had a significant correlation with NoGo errors during the satiety state (Evans et al., 2010; Yin et al., 2016). This finding again demonstrates the relationship between P300 and the inhibition control ability of young smokers. Unfortunately, in our study, the P300 amplitudes and latency of smoker did not significantly change after $12 \mathrm{~h}$ abstinence. This may be caused by a much shorter abstinence time and insufficient sample size. In the future, longer abstinence time and insufficient number of samples can be applied to further address this issue.

\section{Limitations}

First, only young male smokers participated in the experiment and the sample size was in sufficient which may not be a representative sample of young smokers. Second, our experiments did not show changes in the control inhibition ability of young smokers after $12 \mathrm{~h}$ abstinence, which may be due to a much shorter abstinence time. In the future, more samples will be used for future research and female smokers will be added. In addition, a longer abstinence time experiment design will be used for the next study. 


\section{CONCLUSION}

In the present study, we found ERP changes in young smokers after 12-h abstinence. The longer N200 latency and the NoGo N200 latency were correlated with NoGo errors and Go reaction time during the Go/NoGo task. The present findings improved the understanding of the effect of acute smoking abstinence on electrophysiology which may contribute new insights into the neural mechanism of nicotine abstinence.

\section{DATA AVAILABILITY}

All datasets generated for this study are included in the manuscript and/or the supplementary files.

\section{ETHICS STATEMENT}

The procedures of this study were approved by the Medical Ethical Committee of the First Affiliated Hospital of Baotou Medical College, Inner Mongolia University of Science and Technology, and were conducted in accordance with the Declaration of Helsinki. All participants and their legal guardians signed in an informed consent after understanding the purpose of our research.

\section{REFERENCES}

Beaver, J. D., Long, C. J., Cole, D. M., Durcan, M. J., Bannon, L. C., Mishra, R. G., et al. (2011). The effects of nicotine replacement on cognitive brain activity during smoking withdrawal studied with simultaneous fMRI/EEG. Neuropsychopharmacology 36, 1792-1800. doi: 10.1038/npp.2011.53

Bekker, E. M., Kenemans, J. L., and Verbaten, M. N. (2005). Source analysis of the N2 in a cued Go/NoGo task. Cogn. Brain Res. 22, 221-231. doi: 10.1016/ j.cogbrainres.2004.08.011

Bi, Y., Zhang, Y., Li, Y., Yu, D., Yuan, K., and Tian, J. (2017). 12h abstinenceinduced right anterior insula network pattern changes in young smokers. Drug Alcohol Depend. 176, 162-168. doi: 10.1016/j.drugalcdep.2017.02.019

Bokura, H., Yamaguchi, S., and Kobayashi, S. (2001). Electrophysiological correlates for response inhibition in a Go/NoGo task. Clin. Neurophysiol. 112, 2224-2232. doi: 10.1016/s1388-2457(01)00691-5

Borst, G., Cachia, A., Vidal, J., Simon, G., Fischer, C., Pineau, A., et al. (2014). Folding of the anterior cingulate cortex partially explains inhibitory control during childhood: a longitudinal study. Dev. Cogn. Neurosci. 9, 126-135. doi: 10.1016/j.dcn.2014.02.006

Bu, L., Yu, D., Su, S., Yao, M., Deneen, K. M. V., Lin, L., et al. (2016). Functional connectivity abnormalities of brain regions with structural deficits in young adult male smokers. Front. Hum. Neurosci. 10:494. doi: 10.3389/fnhum.2016. 00494

Buzzell, G. A., Fedota, J. R., Roberts, D. M., and Mcdonald, C. G. (2014). The N2 ERP component as an index of impaired cognitive control in smokers. Neurosci. Lett. 563, 61-65. doi: 10.1016/j.neulet.2014.01.030

Cahill, K., Stead, L. F., and Lancaster, T. (2012). Nicotine Receptor Partial Agonists for Smoking Cessation. Hoboken, NJ: John Wiley \& Sons, Ltd.

Cheng, J., Guan, Y., Zhang, Y., Bi, Y., Bu, L., Li, Y., et al. (2016). Electrophysiological mechanisms of biased response to smoking-related cues in young smokers. Neurosci. Lett. 629, 85-91. doi: 10.1016/j.neulet.2016. 06.062

Cid-Fernández, S., Lindín, M., and Díaz, F. (2014). Effects of amnestic mild cognitive impairment on $\mathrm{N} 2$ and $\mathrm{P} 3 \mathrm{Go} / \mathrm{NoGo} \mathrm{ERP}$ components. J. Alzheimers Dis. 38, 295-306. doi: 10.3233/JAD-130677

\section{AUTHOR CONTRIBUTIONS}

DY, KY, and YL conceived and designed the experiments. DX, XW, TX, MZ, and GR performed the experiments. CL, FD, DX, and $\mathrm{XW}$ analyzed the data. CL and FD wrote the manuscript. TX, YR, DY, KY, and KD provided the critical revision of the manuscript. All authors critically reviewed the content and approved the final version for publication.

\section{FUNDING}

This work was supported by the National Natural Science Foundation of China under the Grant Nos. 81571753, 81571751, $81871430,81871426,61771266,81701780,31800926,81401478$, 81470816, and 81471737, the Fundamental Research Funds for the Central Universities under the Grant No. JB151204, the Program for Young Talents of Science and Technology in Universities of Inner Mongolia Autonomous Region NJYT-17B11, the Natural Science Foundation of Inner Mongolia under the Grant Nos. 2017MS(LH)0814 and 2018LH08079, the Program of Science and Technology in Universities of Inner Mongolia Autonomous Region NJZY17262, the Natural Science Basic Research Plan in Shaanxi Province of China under the Grant No. 2018JM7075, and the US National Institutes of Health, Intramural Research Program Y1AA3009.

Donkers, F. C., and van Boxtel, G. J. (2004). The N2 in go/no-go tasks reflects conflict monitoring not response inhibition. Brain Cogn. 56, 165-176. doi: 10.1016/j.bandc.2004.04.005

Evans, D. E., Park, J. Y., Maxfield, N., and Drobes, D. J. (2010). Neurocognitive variation in smoking behavior and withdrawal: genetic and affective moderators. Genes Brain Behav. 8, 86-96. doi: 10.1111/j.1601-183X.2008.00 445.x

Fagerstrom, K. O., and Schneider, N. G. (1989). Measuring nicotine dependence: a review of the Fagerstrom Tolerance Questionnaire. J. Behav. Med. 12, 159-182. doi: $10.1007 /$ bf00846549

Falkenstein, M., Hoormann, J., and Hohnsbein, J. (1999). ERP components in Go/Nogo tasks and their relation to inhibition. Acta Psychol. 101, 267-291. doi: 10.1016/s0001-6918(99)00008-6

Gajewski, P. D., and Falkenstein, M. (2013). Effects of task complexity on ERP components in Go/Nogo tasks. Int. J. Psychophysiol. 87, 273-278. doi: 10.1016/ j.ijpsycho.2012.08.007

Galván, A., Poldrack, R., Baker, C., McGlennen, K., and London, E. (2011). Neural correlates of response inhibition and cigarette smoking in late adolescence. Neuropsychopharmacology 36, 970-978. doi: 10.1038/npp.2010.235

Géczy, I., Czigler, I., and Balázs, L. (1999). Effects of cue information on response production and inhibition measured by event-related potentials. Acta Physiol. Hung. 86, 37-44.

Glenn, S. W., Sinha, R., and Parsons, O. A. (1993). Electrophysiological indices predict resumption of drinking in sober alcoholics. Alcohol 10, 89-95. doi: 10.1016/0741-8329(93)90086-4

Heimstra, N. W., Fallesen, J. J., Kinsley, S. A., and Warner, N. W. (1980). The effects of deprivation of cigarette smoking on psychomotor performance. Ergonomics 23, 1047-1055. doi: 10.1080/00140138008924813

Jacobsen, L. K., Krystal, J. H., Mencl, W. E., Westerveld, M., Frost, S. J., and Pugh, K. R. (2004). Effects of smoking and smoking abstinence on cognition in adolescent tobacco smokers. Biol. Psychiatry 57, 56-66. doi: 10.1016/j.biopsych. 2004.10.022

Jamal, A., Agaku, I. T., O'Connor, E., King, B. A., Kenemer, J. B., and Neff, L. (2014). Current cigarette smoking among adults-United States, 2005-2013. Morb. Mortal. Wkly. Rep. 63, 1108-1112. 
Johnstone, S. J., Pleffer, C. B., Barry, R. J., Clarke, A. R., and Smith, J. L. (2005). Development of inhibitory processing during the Go/NoGo task: a behavioral and event-related potential study of children and adults. J. Psychophysiol. 19, 11-23. doi: 10.1016/j.ijpsycho.2017.10.014

Kai, Y., Meng, Z., Yu, D., Manza, P., Volkow, N. D., Wang, G. J., et al. (2018). Striato-cortical tracts predict 12-h abstinence-induced lapse in smokers. Neuropsychopharmacology 43, 2452-2458. doi: 10.1038/s41386-018-0182-x

Kaiser, S., Weiss, O., Hill, H., Markela-Lerenc, J., Kiefer, M., and Weisbrod, M. (2006). N2 event-related potential correlates of response inhibition in an auditory Go/Nogo task. Int. J. Psychophysiol. 61, 279-282. doi: 10.1016/j. ijpsycho.2005.09.006

Kamarajan, C., and Porjesz, B. (2015). Advances in electrophysiological research. Alcohol Res. Curr. Rev. 37, 53-87.

Kenford, S. L., Smith, S. S., Wetter, D. W., Jorenby, D. E., Fiore, M. C., and Baker, T. B. (2002). Predicting relapse back to smoking: contrasting affective and physical models of dependence. J. Consult. Clin. Psychol. 70, 216-227. doi: 10.1037/0022-006x.70.1.216

Lantz, P. M. (2003). Smoking on the rise among young adults: implications for research and policy. Tob. Control 12, i60-i70.

Lerman, C., Gu, H., Loughead, J., Ruparel, K., Yang, Y., and Stein, E. A. (2014). Large-scale brain network coupling predicts acute nicotine abstinence effects on craving and cognitive function. JAMA Psychiatry 71, 523-530. doi: 10.1001/ jamapsychiatry.2013.4091

Li, Y., Yuan, K., Bi, Y., Guan, Y., Cheng, J., Zhang, Y., et al. (2016). Neural correlates of 12-h abstinence-induced craving in young adult smokers: a resting-state study. Brain Imaging Behav. 11, 677-684. doi: 10.1007/s11682-016-9544-3

Li, Y., Yuan, K., Cai, C., Feng, D., Yin, J., Bi, Y., et al. (2015). Reduced frontal cortical thickness and increased caudate volume within fronto-striatal circuits in young adult smokers. Drug Alcohol Depend. 151, 211-219. doi: 10.1016/j. drugalcdep.2015.03.023

Ling, P. M., Neilands, T. B., and Glantz, S. A. (2009). Young adult smoking behavior: a national survey. Am. J. Prev. Med. 36, 389.e-394.e. doi: 10.1016/j. amepre.2009.01.028

Littel, M., Berg, I. V. D., Luijten, M., Rooij, A. J. V., Keemink, L., and Franken, I. H. (2012). Error processing and response inhibition in excessive computer game players: an event-related potential study. Addict. Biol. 17, 934-947. doi: 10.1111/j.1369-1600.2012.00467.x

Lubman, D. I., Yücel, M., and Pantelis, C. (2015). Addiction, a condition of compulsive behaviour? Neuroimaging and neuropsychological evidence of inhibitory dysregulation. Addiction 99, 1491-1502. doi: 10.1111/j.1360-0443. 2004.00808.x

Luijten, M., Littel, M., and Franken, I. H. A. (2011). Deficits in inhibitory control in smokers during a Go/NoGo task: an investigation using event-related brain potentials. PLoS One 6:e18898. doi: 10.1371/journal.pone.0018898

Oldfield, R. C. (1971). The assessment and analysis of handedness: the Edinburgh inventory. Neuropsychologia 9, 97-113. doi: 10.1016/0028-3932(71)90067-4

Parrott, A. C., Garnham, N. J., Wesnes, K., and Pincock, C. (2015). Cigarette smoking and abstinence: comparative effects upon cognitive task performance and mood state. Hum. Psychopharmacol. Clin. Exp. 11, 391-400. doi: 10.1002/ (sici)1099-1077(199609)11:5<391::aid-hup780>3.3.co;2-q

Parvaz, M. A., Aliaklein, N., Woicik, P. A., Volkow, N. D., and Goldstein, R. Z. (2011). Neuroimaging for drug addiction and related behaviors. Rev. Neurosci. 22, 609-624. doi: 10.1515/RNS.2011.055

Patel, S. H., and Azzam, P. N. (2005). Characterization of N200 and P300: selected studies of the event-related potential. Int. J. Med. Sci. 2, 147-154. doi: 10.7150/ ijms.2.147

Paulsen, D. J., Hallquist, M. N., Geier, C. F., and Luna, B. (2015). Effects of incentives, age, and behavior on brain activation during inhibitory control: a longitudinal fMRI study. Dev. Cogn. Neurosci. 11, 105-115. doi: 10.1016/j.den. 2014.09.003

Su, S., Yu, D., Cheng, J., Chen, Y., Zhang, X., Guan, Y., et al. (2017). Decreased global network efficiency in young male smoker: an EEG study during the resting state. Front. Psychol. 8:1605. doi: 10.3389/fpsyg.2017. 01605

Tian, Y., and Yao, D. (2008). A study on the neural mechanism of inhibition of return by the event-related potential in the Go/Nogo task. Biol. Psychol. 79, 171-178. doi: 10.1016/j.biopsycho.2008.04.006

Wang, C., Bai, J., Wang, C., Deneen, K. M. V., Yuan, K., and Cheng, J. (2017). Altered thalamo-cortical resting state functional connectivity in smokers. Neurosci. Lett. 653, 120-125. doi: 10.1016/j.neulet.2017. 05.038

World Health Organization. (2013). WHO Report on the Global Tobacco Epidemic, 2013. Enforcing Bans on Tobacco Advertising, Promotion and Sponsorship. Geneva: World Health Organization.

Yin, J., Yuan, K., Feng, D., Cheng, J., Li, Y., Cai, C., et al. (2016). Inhibition control impairments in adolescent smokers: electrophysiological evidence from a Go/NoGo study. Brain Imaging Behav. 10, 497-505. doi: 10.1007/s11682-0159418-0

Yu, D., Yuan, K., Bi, Y., Luo, L., Zhai, J., Liu, B., et al. (2017a). Altered interhemispheric resting-state functional connectivity in young male smokers. Addict. Biol. 23, 772-780. doi: 10.1111/adb.12515

Yu, D., Yuan, K., Cheng, J., Guan, Y., Li, Y., Bi, Y., et al. (2017b). Reduced thalamus volume may reflect nicotine severity in young male smokers. Nicot. Tob. Res. 20, 434-439. doi: 10.1093/ntr/ntx146

Yuan, K., Yu, D., Bi, Y., Li, Y., Guan, Y., Liu, J., et al. (2016a). The implication of frontostriatal circuits in young smokers: a resting-state study. Hum. Brain Mapp. 37, 2013-2026. doi: 10.1002/hbm.23153

Yuan, K., Yu, D., Cai, C., Feng, D., Li, Y., Bi, Y., et al. (2016b). Frontostriatal circuits, resting state functional connectivity and cognitive control in internet gaming disorder. Addict. Biol. 22, 813-822. doi: 10.1111/adb.12348

Yuan, K., Yu, D., Bi, Y., Wang, R., Li, M., Zhang, Y., et al. (2017). The left dorsolateral prefrontal cortex and caudate pathway: new evidence for cueinduced craving of smokers. Hum. Brain Mapp. 38, 4644-4656. doi: 10.1002/ hbm. 23690

Yuan, K., Yu, D., Zhao, M., Li, M., Wang, R., Li, Y., et al. (2018). Abnormal frontostriatal tracts in young male tobacco smokers. Neuroimage 183, 346-355. doi: 10.1016/j.neuroimage.2018.08.046

Zhang, Y., Li, M., Wang, R., Bi, Y., Li, Y., Yi, Z., et al. (2017). Abnormal brain white matter network in young smokers: a graph theory analysis study. Brain Imaging Behav. 12, 345-356. doi: 10.1007/s11682-017-9699-6

Zhao, S., Li, Y., Li, M., Wang, R., Bi, Y., Zhang, Y., et al. (2018). 12-h abstinenceinduced functional connectivity density changes and craving in young smokers: a resting-state study. Brain Imaging Behav. 13, 953-962. doi: 10.1007/s11682018-9911-3

Conflict of Interest Statement: The authors declare that the research was conducted in the absence of any commercial or financial relationships that could be construed as a potential conflict of interest.

Copyright $\odot 2019$ Liu, Dong, Li, Ren, Xie, Wang, Xue, Zhang, Ren, von Deneen, Yuan and $Y u$. This is an open-access article distributed under the terms of the Creative Commons Attribution License (CC BY). The use, distribution or reproduction in other forums is permitted, provided the original author(s) and the copyright owner(s) are credited and that the original publication in this journal is cited, in accordance with accepted academic practice. No use, distribution or reproduction is permitted which does not comply with these terms. 